\title{
Ecuaciones constitutivas de la fluencia en caliente de aceros microaleados
}

\author{
J.M. Cabrera ${ }^{(*)}$ y J.M. Prado ${ }^{(*)}$
}

\begin{abstract}
Resumen Durante siglos el conformado en caliente se ha utilizado para proporcionar a los metales la forma deseada. Hoy en día la deformación en caliente no sólo genera la geometría deseada sino las características mecánicas requeridas. Así, la obtención de una ecuación constitutiva para la fluencia en caliente de un acero en particular, es una tarea ineludible para efectuar simulaciones por ordenador de procesos industriales.

La complicación de la modelización de la fluencia a alta temperatura proviene de la simultaneidad de dos fenómenos contrapuestos durante la deformación, a saber, endurecimiento por deformación y ablandamiento por recuperación de la estructura. Este último a su vez puede constar de restauración y recristalización dinámicas.

En este trabajo se revisan y plantean las ecuaciones constitutivas que describen las curvas de fluencia bajo condiciones de deformación en caliente, y se presenta una aplicación de las mismas a aceros microaleados.
\end{abstract}

Palabras clave: Ecuaciones constitutivas. Aceros. Deformación en caliente.

\section{Constitutive equations of the hot flow behaviour of microalloyed steels}

\begin{abstract}
Over centuries, the hot forming has been employed to provide shape to metals. Nowadays, the deformation at high temperatures gives also the required final mechanical properties. From this point of view, the obtention of a constitutive equation describing the hot flow behaviour of microalloyed steels in particular is a very important task.

The main difficulty in modelling the high temperature flow arises from the simultaneity of two opposite phenomena, namely, work hardening and dynamic softening due to recovery and recrystallization.

In this is work a review of constitutive equations (including the effect of the chemical composition) describing the flow behaviour of microalloyed steels deformed under high temperature conditions.
\end{abstract}

Keywords: Constitutive equations. Steels. Hot working.

\section{INTRODUCCIÓN}

Los procesos involucrados en la deformación de materiales metálicos son el resultado de dos fenómenos competitivos $(1,2)$, un balance entre mecanismos de aumento de resistencia (endurecimiento por deformación) y de ablandamiento (restauración y recristalización dinámicas). Por eso la curva de fluencia alcanza un estado de saturación cuando ambos mecanismos se compensan (ver figura 1). Si ocurre la recristalizacion dinámica, una vez alcan-

(*) Dpto. de Ciencia de Materiales e Ingeniería Metalúrgica, ETSEIB-Univ. Politécnica de Catalunya. Av. Diagonal 647, 08028-Barcelona (España). zado un máximo en la curva de fluencia se produce un ablandamiento adicional.

La modelización de las curvas de fluencia puede efectuarse por etapas. Hasta el valor máximo de tensión, definido por su deformación asociada $\varepsilon_{p}$, se puede suponer que sólo operan el endurecimiento por deformación y la restauración dinámica, pudiendo utilizarse, para este caso, modelos de base física. En cambio, el empleo de modelos teóricos para la recristalización dinámica es todavía una tarea en discusión. Este inconveniente se puede solventar suponiendo que las fracciones de volumen recristalizadas son proporcionales al ablandamiento de la curva $\sigma-\varepsilon$, y estudiando entonces la cinética de la recristalización dinámica. 


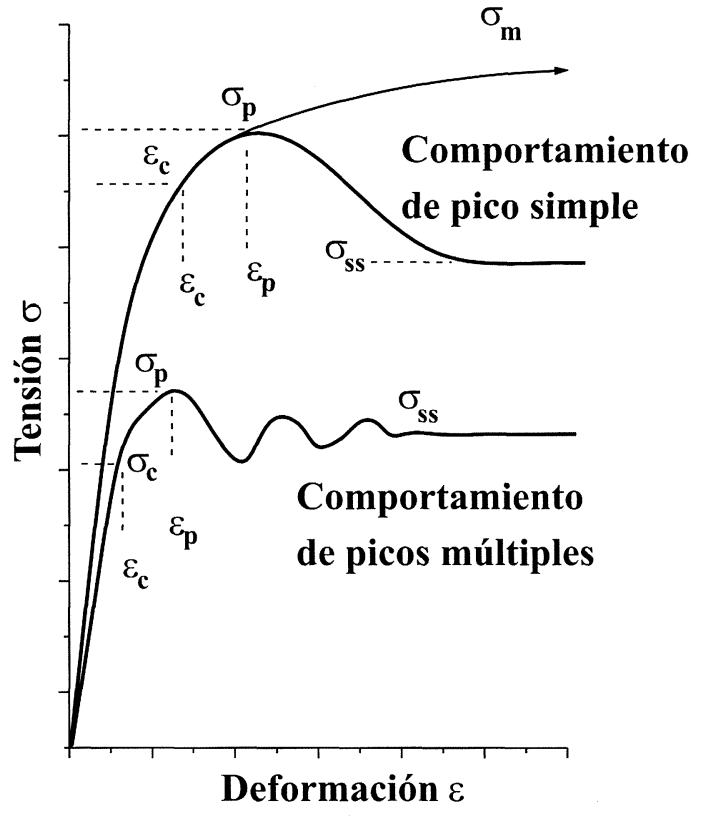

FIG. 1.- Representación esquemática de las curvas de fluencia en caliente de un acero.

FIG. 1.- Representation of flow curves of an austenitic steel under high temperature conditions.

\section{CARACTERIZACIÓN DEL INICIO DE LA RECRISTALIZACIÓN DINÁMICA}

En primer lugar hay que determinar una ecuación cinética para la deformación crítica de recristalización $\varepsilon_{p}$. Ello es imprescindible para saber a partir de qué valor de deformación $\varepsilon$ y en qué condiciones de temperatura $T$ y velocidad de deformación $\varepsilon$ es necesario considerar la recristalización dinámica. Experimentalmente (3) se verifica que:

$$
\varepsilon_{p}=K_{\varepsilon} \cdot d_{0}^{n_{\varepsilon}} \cdot Z^{m_{\varepsilon}}
$$

donde $d_{0}$ es el tamaño de grano inicial, $Z$ el parámetro de Zener-Hollomon y $K_{\varepsilon}, n_{\varepsilon}$ y $m_{\varepsilon}$ son constantes del material que pueden depender de la composición química.

En la tabla I se muestran las constantes de la ec. [1] según varios autores (4-13) y para diferentes aceros, apreciándose cierta discrepancia entre los diversos investigadores. Esto puede deberse a varios motivos: i) energías de activación aparente $Q$ diferentes, ii) exponente de tamaño de grano arbitrariamente fijado en 0,5 , iii) el método de ensayo empleado (torsión o compresión producen diferencias en la determinación de $\varepsilon_{p}$ (14), y iv) diferente dependencia con la composición química de las constantes (un elemento microaleante modifica su carácter como retardador del inicio de la recristalización dinámica si está disuelto o precipitado).
TABLA I.- Recopilación de constantes en la ec. [1] reportadas en bibliografía

TABLE I.- Summary of values reported in the literature for the constants in eq. [1]

\begin{tabular}{|c|c|c|c|c|c|}
\hline Acero & Ref & $K_{\varepsilon}\left(10^{4}\right)$ & $n_{\varepsilon}$ & $m_{\varepsilon}$ & $Q \mathrm{~kJ} / \mathrm{mol}$ \\
\hline C-Mn-Al & 4 & 5,5 & 0,5 & 0,15 & 270 \\
& & & & & 290 \\
C-Mn & 5 & 7 & 0,3 & 0,17 & 312 \\
C & 6 & 4,9 & 0,5 & 0,15 & 312 \\
C & 6 & 3,7 & 0,44 & 0,19 & 312 \\
C-Mn-V & 7 & - & - & 0,11 & - \\
C-Mn-Nb & 7 & - & - & 0,23 & - \\
C-Mn & 8 & 5,4 & 0,5 & 0,16 & 310 \\
C-Mn-Ti & 9 & 1,25 & 0,75 & 0,18 & 315 \\
C-Mn & 10 & 4,2 & 0,5 & 0,14 & 300 \\
C-Mn & 11 & 27 & 0,20 & 0,15 & - \\
C-Mn-Si & 12 & 90 & 0,19 & 0,15 & 272 \\
C-Mn-Si & 13 & 70 & 0,15 & 0,14 & 270 \\
\hline
\end{tabular}

Notas: Los aceros son de bajo C excepto los de las refs. 4, 11 y 13 que son de medio $\mathrm{C}$, y el de la ref. 10 que es de alto $\mathrm{C} y$ aleado con Ni. La ref. 8 es para aceros resulfurados. En la ref. 12 se incluye un término adicional al parámetro de Zener-Hollomon que a su vez depende de la composición química y de la energía de activación. En las refs. 12 y 13 los aceros son multimicroaleados.

No es usual encontrar en la bibliografía estudios sobre la dependencia de las anteriores constantes con la composición química. Una notable excepción es el estudio de Medina y col. (12) en el que se determina la citada dependencia por familias de aceros. En ese estudio, donde los elementos microaleantes están en disolución, se aprecia que, en el rango de composiciones estudiado (bajo carbono), el C, Mn y Si no tienen una influencia significativa sobre $\varepsilon_{p}$ mientras que el orden de influencia de los microaleantes es $\mathrm{V}, \mathrm{Ti}$, Mo y $\mathrm{Nb}$, en consonancia con resultados parciales de otros autores (15-20).

\section{FORMALISMO MECÁNICO DE LA CURVA DE FLUENCIA HASTA LA TENSIÓN MÁXIMA}

Durante la deformación en caliente se acepta que la densidad de dislocaciones móviles $\rho$ evoluciona con la deformación del siguiente modo (1, 21-25):

$$
\frac{\partial \rho}{\partial \varepsilon}=\left.\frac{\partial \rho}{\partial \varepsilon}\right|_{\text {almacenadas }}-\left.\frac{\partial \rho}{\partial \varepsilon}\right|_{\text {restauradas }}
$$


El primer término es el responsable del endurecimiento por deformación, mientras que el segundo lo es de los procesos de recuperación o reordenamiento de las dislocaciones previamente almacenadas. Asimismo, está bien establecido que la tensión $\sigma$ debida al deslizamiento de las dislocaciones está relacionada con $\rho$ según:

$$
\sigma=\alpha^{\prime} \mu b \sqrt{\rho}
$$

donde $b$ es el vector de Burgers, $\alpha^{\prime}$ es una constante de proporcionalidad y $\mu$ es el módulo de cizalladura. La combinación de las ecs. [2] y [3] con la ley de velocidad de endurecimiento

$$
\theta=\left.\left(\frac{\partial \sigma}{\partial \varepsilon}\right)\right|_{\dot{\varepsilon}, T}
$$

permite predecir la evolución de $\rho$ con $\varepsilon$, y por lo tanto de $\sigma$, esto es, el comportamiento a fluencia.

La resolución de las ecuaciones anteriores pasa por determinar la evolución de $\theta$ con $\sigma$. Se han propuesto diversos modelos en la bibliografía (21-25), pero es el modelo de Estrin y Mecking (23) y Bergström $(24,25)$ el que proporciona resultados más satisfactorios. Estrin y Mecking afirman que el camino libre medio de las dislocaciones es una constante geométricamente impuesta, y por lo tanto la velocidad de almacenamiento de dislocaciones, proporcional al citado camino, es también constante. Además, suponen que la restauración dinámica sigue una cinética de primer orden (lineal). Estas hipótesis transforman la ec. [2] en la siguiente expresión:

$$
\left(\frac{\partial \rho}{\partial \varepsilon}\right)=U-\Omega \rho
$$

La integración de la ec. [4] es relativamente sencilla si se acepta que $U$ y $\Omega$ son independientes de $\varepsilon$. Con esta presunción y tomando como condición inicial $\rho=\rho_{0}$ para $\varepsilon=0$ resulta:

$$
\rho=\rho_{0} e^{-\Omega \varepsilon}+\frac{U}{\Omega}\left(1-e^{-\Omega \varepsilon}\right)
$$

Introduciendo la ec. [3] en la anterior expresión se obtiene la ecuación de modelización:

$$
\sigma=\left[\sigma_{0}^{2} e^{-\Omega \varepsilon}+\left(\alpha^{\prime} \mu b\right) \frac{U}{\Omega}\left(1-e^{-\Omega \varepsilon}\right)\right]^{0,5}
$$

o bien:

$$
\sigma=\left[\sigma_{m}^{2}+\left(\sigma_{0}^{2}-\sigma_{m}^{2}\right) e^{-\Omega \varepsilon}\right]^{0,5}
$$

donde:

$$
\sigma_{0}=\alpha^{\prime} \mu b \sqrt{\rho_{0}}
$$

y

$$
\sigma_{m}=\alpha^{\prime} \mu b \sqrt{\frac{U}{\Omega}}
$$

siendo $\sigma_{m}$ la tensión de saturación de la curva de fluencia en ausencia de recristalización dinámica. En el caso de aparición de recristalización dinámica puede suponerse en primera aproximación que $\sigma_{p}=$ $\sigma_{m}$. Para que las ecs. [6] o [7] sean verdaderas ecuaciones constitutivas, es necesario determinar el efecto de $\dot{\varepsilon}, T$ y la composición química en los valores de $\sigma_{m}, U$ y $\Omega$.

\section{ECUACIÓN CINÉTICA PARA $\sigma_{m}$}

La dependencia de $\dot{\varepsilon}$ con $\sigma_{m}$ (o $\sigma_{p}$, e incluso $\sigma_{s s}$ ) durante el conformado en caliente, puede explicarse por las ecuaciones siguientes $(2,21,26)$ :

$$
\begin{gathered}
\dot{\varepsilon}=A^{\prime} \cdot \sigma_{m}^{n} \cdot \exp \left(-\frac{Q}{R T}\right) \\
\dot{\varepsilon}=A^{\prime \prime} \cdot \exp \left(\beta \cdot \sigma_{m}\right) \cdot \exp \left(-\frac{Q}{R T}\right)
\end{gathered}
$$

En estas expresiones $A^{\prime}, A^{\prime \prime}$ y $\beta$ son constantes del material, $n$ es el exponente de "creep", $Q$ es una energía de activación y $R$ la constante de los gases. La ec. [10] es válida a bajas tensiones mientras que la ec. [11] refleja mejor el comportamiento a altas tensiones. Garofalo (27) y Sellars y McTegart (28) propusieron una expresión más general que abarcase ambos intervalos de tensiones:

$$
\dot{\varepsilon}=A^{\prime \prime \prime}\left[\operatorname{senh}\left(\alpha \sigma_{m}\right)\right]^{n} \cdot \exp \left(-\frac{Q}{R T}\right)
$$

donde $A^{\prime \prime \prime}$ es una constante del material y $\alpha$ la tensión inversa que marca el cambio de comportamiento potencial a exponencial.

En la bibliografía se aprecia una aparente divergencia entre los valores reportados en aceros para las constantes $A^{\prime \prime \prime}, \alpha, n$ y $Q$. Así, es frecuente observar que $\alpha$ es una constante cercana a 0,012 $\mathrm{MPa}^{-1}, Q$ depende de la composición química, y $\mathrm{n}$ oscila alrededor de 5 .

En el caso de $Q$ pocos autores han propuesto alguna expresión para tal dependencia. Entre ellos se pueden citar aquí una que tiene en cuenta los elementos microaleantes (29) y otra sólo los elementos de aleación (30): 


$$
\begin{gathered}
Q(\mathrm{~kJ} / \mathrm{mol})=267-2,5(\% \mathrm{C})+1,0(\% \mathrm{Mn})+ \\
+33,6(\% \mathrm{Si})+35,6(\% \mathrm{Mo})+93,7(\% \mathrm{Ti})^{0,59}+ \\
+31,6(\% \mathrm{~V})+70,7(\% \mathrm{Nb})^{0,56} \\
Q(\mathrm{~kJ} / \mathrm{mol})=282,7+92,4(\% \mathrm{C})+ \\
+6,57((\% \mathrm{Mn})+(\% \mathrm{Si}))
\end{gathered}
$$

Otro planteamiento (similar al empleado en "creep") propuesto por los presentes autores $(13,31,32)$ muestra que las discrepancias en las constantes anteriores se reducen cuando $\sigma$ se normaliza por el módulo de Young en función de la temperatura $E(T)$, y $\dot{\varepsilon}$ por el coeficiente de autodifusión $D_{s d}(T)$. Esto equivale a suponer $Q$ independiente de la composición química, lo cual es consistente con la expresión propuesta por Medina y col. (29), puesto que si los elementos de aleación y microaleación están en los porcentajes habituales, $Q$ es muy similar a la energía de activación de autodifusión $Q_{s d}(270 \mathrm{~kJ} / \mathrm{mol})$.

$$
\frac{\dot{\varepsilon}}{D_{s d}(T)}=A\left(\operatorname{senh} \alpha\left(\frac{\sigma_{m}}{E(T)}\right)\right)^{n}
$$

Acorde con los mecanismos de deformación operantes, $n$ puede igualarse a 5 , y entonces sólo son necesarios dos parámetros para la definición de la ecuación cinética, esto es $A$ y $\alpha$. En el caso de que $d_{0}$ sea muy fino $(<30 \mu \mathrm{m})$ ha de considerarse un término adicional de tensión en la anterior ecuación $(13,31,32)$.

En un trabajo paralelo (33), se analizó la anterior expresión para $\sigma_{p}$ con resultados reportados del comportamiento a deformación en caliente de 41 aceros microaleados de medio y bajo carbono, y aceros al C-Mn-Si. En todas las situaciones las condiciones de ensayo aseguraban que los elementos microaleantes estuviesen en solución y que el tamaño de grano inicial no fuese excesivamente fino. Mediante un estudio estadístico se correlacionó la composición química con los valores de $A^{1 / 5}$ y $\alpha$, encontrándose que la expresión que mejor explicaba los resultados experimentales era (\% atómico):

$$
\begin{gathered}
A^{1 / 5}=3,4 \cdot \% \mathrm{Fe}+293 \cdot \% \mathrm{C}-88 \cdot \% \mathrm{Mn}+ \\
+17 \cdot \% \mathrm{Si}-838 \cdot \% \mathrm{~V}-770 \cdot \% \mathrm{Ti}+ \\
+5375 \cdot \% \mathrm{Al}-1920 \cdot \% \mathrm{Nb}-2391 \cdot \% \mathrm{Mo} \\
\alpha=17,1 \cdot \% \mathrm{Fe}-234 \cdot \% \mathrm{C}+201 \cdot \% \mathrm{Mn}- \\
-90 \cdot \% \mathrm{Si}+1410 \cdot \% \mathrm{~V}-694 \cdot \% \mathrm{Ti}- \\
-5149 \cdot \% \mathrm{Al}+1073 \cdot \% \mathrm{Nb}+2755 \cdot \% \mathrm{Mo}
\end{gathered}
$$

Puede apreciarse que un elemento que aumenta $A$, disminuye $\alpha$ y viceversa. Esta generalización aparentemente falla con el Ti. Asimismo, el efecto de los elementos microaleantes sobre $A$ y $\alpha$ es más fuerte que el del C, Mn y Si. El orden creciente de influencia sobre $\alpha$ es: $\mathrm{Al}, \mathrm{Ti}, \mathrm{C}, \mathrm{Si}, \mathrm{Mn}, \mathrm{V}, \mathrm{Nb}$ y Mo. En el caso de $A$, el orden es: Mo, Nb, V, Ti, $\mathrm{Mn}, \mathrm{Si}, \mathrm{C}$ y Al. Nótese que un aumento de $\mathrm{C}$ y Si y de los microaleantes Al y Ti disminuyen el valor de $\alpha$, y por lo tanto extiende la validez de la ley potencial a mayores tensiones. Lo contrario ocurre con el $\mathrm{Mn}$ y el Mo, $\mathrm{Nb}$ y V. Cabe finalmente comentar que es de esperar un comportamiento similar sobre el valor de tensión $\sigma_{s s}$ lo cual se mostrará en breve por los presentes autores (34).

\section{ECUACIONES CINÉTICAS PARA $U$ Y $\Omega$}

Es también inusual encontrar estudios en los que se haga un análisis exhaustivo sobre el efecto de $T$, $\dot{\varepsilon}$ y la composición química, sobre los términos responsables del endurecimiento por deformación y ablandamiento por restauración dinámica.

Según Estrin y Mecking, el parámetro $U$ debe considerarse constante. Los presentes autores, en un estudio de dos aceros microaleados de medio carbono (35) encontraron que $U$ dependía ligeramente de las condiciones de conformado y propusieron la siguiente expresión:

$$
(a b)^{2} U=K_{U} \cdot d_{0}^{n_{U}} \cdot Z^{m_{U}}
$$

Se puede apreciar en la tabla II que el efecto del tamaño de grano sobre el término de endurecimiento (exponente $n_{U}$ ) es mayor que el del parámetro de Zener-Hollomon (exponente $m_{U}$ ). Esto es consistente con la idea de Estrin y Mecking del camino libre medio impuesto geométricamente. Es también evidente que los exponentes $n_{U}$ y $m_{U}$ asociados al acero $V$ son ligeramente mayores que los del acero $M$, lo cual puede deberse a que está más aleado (mayores cantidades de $\mathrm{C}, \mathrm{Mn}$ y $\mathrm{Si}$ ), por lo que es de esperar un mayor efecto de endurecimiento por solución sólida. Por contra, la constante $K_{U}$ parece relativamente insensible a la composición química.

Por lo que hace referencia al término responsable del ablandamiento debe esperarse que sea dependiente del parámetro de Zener-Hollomon ya que la restauración dinámica es un fenómeno activado térmicamente. En la bibliografía se pueden encontrar algunas expresiones de la siguiente forma para $\Omega(36,37)$ :

$$
\Omega=K_{\Omega} \cdot d_{0}^{n_{\Omega}} \cdot Z^{m_{\Omega}}
$$

donde $K_{\Omega}, n_{\Omega}$ y $m_{\Omega}$ son constantes dependientes del material. Los valores determinados por los presentes autores para los dos aceros anteriores se comparan en la tabla III con los de otros trabajos. Puede 
TABLA II.- Valores de los coeficientes que afectan al término de endurecimiento por deformación (ec. [14]) para dos aceros microaleados de medio C

TABLE II.- Values of the coefficients affecting the work hardening term in eq. [14] for two medium carbon microalloyed steels

\begin{tabular}{|c|c|c|c|c|}
\hline Acero & Ref & $K_{U}$ & $n_{U}$ & $m_{U}$ \\
\hline Acero V & 35 & $5,07 \cdot 10^{-6}$ & $-0,217$ & 0,138 \\
Acero M & 35 & $5,43 \cdot 10^{-6}$ & $-0,183$ & 0,126 \\
\hline
\end{tabular}

Nota: Los aceros (Ref. 35) son de contenido medio de carbono.

apreciarse que no hay un excesivo acuerdo en los valores de las constantes. Parte de la discrepancia puede provenir de las diferentes energías de activación empleadas. Un análisis más profundo de los aceros $V$ y $M$ mostró sin embargo que los valores de $\Omega$ de ambos aceros eran similares. No debe olvidarse que los elementos aleantes pueden tener efectos opuestos sobre la restauración dinámica. En consecuencia, y para los dos aceros anteriores, la disminución simultánea de Mn y Si no se notaría si tienen efectos opuestos en el mecanismo de ablandamiento. Cabe mencionar que Herman y col. (39) proponen para aceros al C-Mn que sólo la constante $K_{\Omega}$ es dependiente de la composición química y en concreto exclusivamente dependiente del contenido en $C\left(K_{\Omega}=112,7(1+3,88 \% C)\right)$. Según esta expresión a mayor cantidad de $\mathrm{C}$ más se facilita la restauración dinámica.

\section{LA CINÉTICA DE LA RECRISTALIZACIÓN DINÁMICA}

A la hora de modelizar la curva de fluencia es posible evitar la falta de conocimiento de una ecuación evolutiva para la recristalización dinámica $(3,21)$. Este fenómeno, como todo proceso de transformación de estado sólido que tiene lugar por nucleación y crecimiento, tiene una cinética que puede expresarse por la ec. de Avrami:

$$
X=1-\exp \left(-B^{\prime} t^{k^{\prime}}\right)
$$

donde $X$ es la fracción de volumen recristalizada en un tiempo dado $t$, y $B^{\prime}$ y $k^{\prime}$ son constantes asociadas con el mecanismo y geometría de la nucleación $\left(k^{\prime}\right)$ y con la velocidad de nucleación y crecimiento $\left(B^{\prime}\right)$. Es habitual definir la cinética de la recristalización en términos del tiempo para el $50 \%$ de recristalización $t_{50 \%}(40)$. Puesto que exp $(-0,693)=0,5$ la ec. [16] se transforma en:
TABLA III.- Valores de las constantes que afectan al término de restauración dinámica (ec. [15]) para algunos aceros reportados en bibliografía

TABLE III.- Values of the constants affecting the dynamic recovery term in eq. [15] for different steels, according to results reported in the literature

\begin{tabular}{|c|c|c|c|c|c|}
\hline Acero & Ref & $K_{\Omega}$ & $n_{\Omega}$ & $m_{\Omega}$ & $Q(\mathrm{~kJ} / \mathrm{mol})$ \\
\hline Acero V & 35 & 102 & $-0,20$ & $-0,07$ & 270 \\
Acero M & 35 & 394 & $-0,28$ & $-0,12$ & 270 \\
HSLA & 36 & 157 & - & $-0,2$ & 137 \\
C & 37 & 37,2 & 0,04 & $-0,098$ & 175 \\
C-Mn-Si & 38 & - & - & 0,059 & $272-322$ \\
C-Mn & 39 & - & $-0,2$ & $-0,105$ & 177 \\
\hline
\end{tabular}

Nota: Todos los aceros son de bajo contenido de carbono excepto el de la ref. 35 que es de contenido medio. Todos los aceros excepto los de las refs. 37 y 39 son microaleados. En la ref. $39 K_{\Omega}$ depende de la composición química (ver texto).

$$
X=1-\exp \left(-0,693\left(\frac{t}{t_{50 \%}}\right)^{k^{\prime}}\right)
$$

Experimentalmente se ha comprobado $(41,42)$ que $t_{50} \%$ depende de las condiciones de deformación $(T$ y $\dot{\varepsilon})$ y de la propia estructura o tamaño de grano inicial $d_{0}$ según:

$$
t_{50 \%}=B^{\prime \prime} \cdot \dot{\varepsilon}^{m_{t}} \cdot d_{0}^{n_{t}} \cdot \exp \left(\frac{Q_{\mathrm{rec}}}{R T}\right)
$$

donde $B^{\prime \prime}, m_{t}$ y $n_{t}$ son constantes y $Q_{\text {rec }}$ es una energía de activación. Sabiendo que los ensayos se realizan a $\dot{\varepsilon}$ constante, la ec. [17] se transforma en:

$$
X=1-\exp \left(-0,693 \cdot\left[\frac{\varepsilon-\varepsilon_{p}}{\varepsilon_{50 \%}-\varepsilon_{p}}\right]^{k^{\prime}}\right)
$$

Suponiendo que las fracciones de volúmenes transformadas son proporcionales a los ablandamientos, es posible deducir, a partir de las curvas de fluencia, la cinética de la transformación y por lo tanto, expresar la evolución de $\sigma$ con $\varepsilon$ desde la tensión máxima $\sigma_{p}$ hasta la de estado estable $\sigma_{s s}$ siguiendo una expresión similar a la de Avrami:

$$
\begin{gathered}
\sigma=\sigma_{p}-\left(\sigma_{p}-\sigma_{s s}\right) . \\
{\left[1-\exp \left(-0,693 \cdot\left[\frac{\varepsilon-\varepsilon_{p}}{\dot{\varepsilon} \cdot t_{50 \%}}\right]^{k^{\prime}}\right)\right]}
\end{gathered}
$$

ecuación que sólo es válida para $\varepsilon>\varepsilon_{p}$. 
Tradicionalmente, el exponente $k^{\prime}$ se considera constante e independiente de las condiciones de ensayo, lo cual es cierto si no hay cambios en los mecanismos de nucleación $(40,43)$. Los valores más habituales para aceros $(21,36,38,44-49)$ oscilan entre 1 y 2 (ver tabla IV). Estos valores apoyan la evidencia experimental de nucleación en límites y aristas de grano. Cabe notar que algunos autores han encontrado que el exponente de Avrami depende del parámetro de Zener-Hollomon (45,50) (lo que ayuda a explicar la transición entre recristalización dinámica de pico simple y continua (50)), y además puede depender de la composición química (38).

Han podido verificarse en la bibliografía $(13,42,47,48)$ algunos resultados para $t_{50} \%$ o bien para $\varepsilon_{50 \%}$ (equivalente a $t_{50 \%}$ sin más que dividir por $\varepsilon$ ), como se recoge en la tabla V. Se aprecia que el exponente del tamaño de grano es similar en todos los aceros, habiendo discrepancias en la constante $B^{\prime \prime}$, en las energías de activación, y en el exponente de $\varepsilon$, lo que hace suponer que estos parámetros son efectivamente dependientes de la composición química.

\section{CONCLUSIONES}

El uso de las ecs. [7] y [20] junto con las ecuaciones cinéticas [1], [13], [14], [15] y [18] permite modelizar la curva de fluencia, haya o no recristalización dinámica. Aunque cada vez están más claros los efectos de la composición química sobre las curvas de fluencia, hay que destacar que todavía son necesarios estudios adicionales para una mejor caracterización de su influencia sobre los parámetros responsables del endurecimiento por deforma-

TABLA IV.- Constantes $k^{\prime}$ para la cinética de recristalización dinámica de aceros

TABLE IV.- Constants $k^{\prime}$ for the kinetics of dynamic recristallization

\begin{tabular}{|c|c|c|}
\hline Acero & Ref & $K^{\prime}$ \\
\hline AISI 304 & 21 & 1,6 \\
C & 44 & 1,4 \\
C (mic) & 36 & $1,5-1,6$ \\
C (mic)* & 45 & $0,7-1,6$ \\
AISI 301, 304, 316, 317 & 46 & $1,2-1,3$ \\
C-Mn & 47 & 2 \\
C-Mn & 48 & 2 \\
AISI 304 & 49 & 1,25 \\
C-Mn-Si (mic) & 38 & $1-3,2$ \\
C-Mn-Si** (mic) & 13,14 & 1,83 \\
\hline
\end{tabular}

TABLA V.- Constantes para la determinación de $t_{50 \%}$ (ec. [18])

TABLE V.- Constants in eq [18] to determine $t_{50 \%}$

\begin{tabular}{|c|c|c|c|c|c|}
\hline Acero & Ref & $B^{\prime \prime}$ & $n_{t}$ & $-m_{t}$ & $Q_{\text {red }} / R$ \\
\hline C-Mn-Si & 13 & $1.8 \cdot 10^{-6}$ & 0,24 & 0,75 & 13.974 \\
C-Mn & 42 & $1,0 \cdot 10^{-5}$ & - & 0,6 & 14.440 \\
C-Mn & 47 & $6,9 \cdot 10^{-4}$ & 0,27 & 0,96 & 7.190 \\
C-Mn-Si & 48 & $1,1 \cdot 10^{-3}$ & 0,28 & 0,95 & 6.420 \\
\hline
\end{tabular}

Nota: Los aceros son de bajo $\mathrm{C}$ excepto el de la ref. 13 que es de medio C. En la ref. 42 el exponente de la ecuación de Avrami depende de las condiciones de deformación.

ción $U$, el ablandamiento por restauración dinámica $\Omega$, y los de la cinética de recristalización dinámica $k^{\prime}$ y $t_{50 \%}$.

\section{Agradecimiento}

La financiación de este trabajo se realizó mediante un proyecto CICYT ref. MAT 97-0827C02-01.

\section{REFERENCIAS}

(1) Blum, W. en Materials Science and Technology, 6, Ed. VCH, Weinheim, 1991: 363-405.

(2) Frost, H.J. y Ashby, M.F. en Deformation-mechanism maps, Ed. Pergamon Press, Oxford, 1982: 1-30.

(3) SAKAI, T. y JonAS, J.J. Acta Metall., 32 (2), 1984: 189209.

(4) Mintz, B., Jonas, J.J. y Abu-shosa, R. Mater. Sci. Technology, 7, 1991: 904-913.

(5) Sellars, C.M. Mater. Sci. and Technology, 6, 1990: 1072-1081.

(6) Devadas, C., Samarasekera, I.V. y Hawbolt, E.B. Metall. Trans., 22A, 1991: 335-349.

(7) Pussegoda, L.N., Hodgson, P.D. y Jonas, J.J. Mater. Sci. and Technology, 8, 1992: 63-71.

(8) Nazabal, J.L., Urcola, J.J. y Fuentes, M. Metals Technology, 9, 1982: 323-326.

(9) Ruibal, E., Urcola, J.J. y Fuentes, M. Metals Technology, 11, 1984: 189-195.

(10) San Martin, G.E., Nuñez Pettinari, S.I., Ruzzante, J.E. y Merlone, G.F. Scripta Metall. et Mater., 26, 1992: 405-410.

(11) ANELLI, C. ISIJ International, 32 (3), 1992: 440-449.

(12) Medina, S.F. y Hernandez, C.A. Acta Metall. Mater. 44, 1996: 149-154.

(13) Cabrera, J.M., Al Omar, A., Jonas, J.J. y Prado, J.M. Metall. Trans., 28A, 1997: 2233-2245.

(14) Weiss, I., Sakai, T. y Jonas, J.J. Metal Science, 18, 1984: 77-84. 
(15) Cuddy, L.D. en Thermomechanical Processing of Microalloyed Austenite, Eds. A.J. DeArdo, G.A. Ratz, P.J. Wray. Metallurgical Society of AIME, Pittsburg, 1981: 129-139.

(16) Akben, M.G., Weiss, I. y Jonas, J.J. Acta Metall., 29, 1981: 111-121.

(17) Jonas, J.J. y Akben, M.G. Metals Forum, 4, nº1\&2, 1981: 92-101.

(18) Akben, M.G., Chandra, T., Plassiard, P. y Jonas, J.J. Acta Metall., 32, 1984: 591-601.

(19) Jonas, J.J. y WeISS, I. Metals Science, 1979: 238-245.

(20) Weiss, I. y Jonas, J.J. Metall. Trans., 10A, 1979: 831840.

(21) Roberts, W. en Deformation, Processing and Structure, Ed. G. Krauss, ASM, Ohio, 1982: 109-184.

(22) KоскS, U.F. Engineering Materials and Technology, Trans. ASME, 98, 1976: 76-85.

(23) Estrin, Y. y Mecking, H. Acta Metall., 32 (1), 1984: 57 70.

(24) Bergström, Y. Mater. Sci. Engineering, 5, 1969-1970: 193-200.

(25) Bergström, Y. y Aronsson, B. Metall. Trans., 3, 1972: 1951-1957.

(26) Jonas, J.J., Sellars, C.M. y Tegart, W.J. McG. Metallurgical Reviews, 14, 1969: 1-12.

(27) Garofalo, F. Transactions of AIME, 227, 1963: 351356.

(28) Sellars, C.M. y Tegart, W.J. McG. Mém. Sci. de la Revue de Metallurgie, LXIII (9), 1966: 731-746.

(29) Medina, S.F. y Hernandez, C.A. Acta Metall. Mater. 44, 1996: 137-148.

(30) Hinojosa, M., Ortiz, U. y Colas, R. Mat. Science Forum, 113-115, 1993: 467-472.

(31) Cabrera, J.M., Jonas, J.J. y Prado, J.M. Mat. Sci. and Technology, 12, 1996: 579-585.

(32) Cabrera, J.M., Al Omar, A., Jonas, J.J. y Prado, J.M., en Fundamentals and Applications of Microalloying Forging Steels. Eds. Van Tyne, C.J., Krauss, G., Matlock, D.K., TMS, Warrendale, 1996: 225-239.

(33) Cabrera, J.M., Al Omar, A., Jonas, J.J. y Prado, J.M., en Proceedings of ReX'96. The Third International Conference on Recrystallization and Related Phenomena. Ed. Terry R. McNelley, Monterey Institute of Advanced Studies, Monterey, California (USA), 1997: 373-379.

(34) CABReRA, J.M., JonAS, J.J. y Prado, J.M., a presentar en Microalloying in steels: New trends for the $21^{\text {st }}$ century,
International Symposium, 7-9 Septiembre, San Sebastián, 1998.

(35) Cabrera, J.M., Al Omar, A., Jonas, J.J. y Prado, J.M. International Conference on Thermomechanical Processing of Steels and Other Metals, THERMEC'97. July 7-11, Wollongong, Australia, 1997: En imprenta.

(36) LaAsraoui, A. y Jonas, J.J. Metall. Trans., 22A, 1991: 1545-1558.

(37) Yoshie, A., Morikawa, H., Onoe, Y. y Itoh, K. Transactions ISIJ, 27, 1987: 425-431.

(38) Hernandez, C.A., Medina, S.F. y Ruiz, J. Acta Metall. Mater. 44, 1996: 155-171.

(39) Herman, J., Thomas, B. y LotTer, U., en Technical Steel Research (Final Report), European Comission EUR 16795 EN, 1997.

(40) Porter, D.A. y Easterling, K.E. en Phase Transformations in Metals and Alloys, Ed. Van Nostrand Reinhold, Berkshire, 1981: Caps. 3 y 5.

(41) Sellars, C.M. Mater. Sci. Technology, 6, 1990: 10721081.

(42) Devadas, C., Samarasekera, I.V. y Hawbolt, E.B. Metall. Trans., 22A, 1991: 335-349.

(43) Christian, J.W. en The Theory of Transformations in Metals' and Alloys, Ed. Pergamon Press, Oxford, 1981: Caps. 1 y 12.

(44) Beynon, J.H. y Sellars, C.M. ISIJ International, 32 (3), 1992: 359-367.

(45) Roucoules, C. Tesis Doctoral, McGill University, Montreal, Canadá, 1992.

(46) McQueen, H.J., Ryan, N.D. Y Evangelista, E. Materials Science Forum, 113-115, 1993: 435-440.

(47) Nanba, S., Kitamura, M., Shimada, M., Katsumata, M., Inone, T., Imamura, H., Maeda, Y. y Hattori, S. ISIJ International, 32 (3), 1992: 377-386.

(48) Anan, G., Nakajima, S., Miyahara, M., Nanba, S., Umemoto, M., Hiramatsu, A., Moriya, A. y Watanabe, T. ISIJ International, 32 (3), 1992: 261-266.

(49) Roberts, W., Bodén, H. y Ahlblom, B. Metal Science, 1979: 195-205.

(50) Cabrera, J.M., Al Omar, A., Jonas, J.J. y Prado, J.M., en Proceedings of ReX'96. The Third International Conference on Recrystallization and Related Phenomena. Ed. Terry R. McNelley, Monterey Institute of Advanced Studies, Monterey, California (USA), 1997: 381-388. 